\title{
A Word from the Guest Editor
}

A considerable amount of research of various types has been conducted around formulaic language-multiword units with unitary meanings or functions that appear to be prefabricated and mentally stored and processed as if single words. As the nature of formulaic language and its use and acquisition have been studied for many years, it is remarkable that there have been so few investigations into how to actually teach this essential element of language to second language (L2) learners. We know that only very advanced learners reach a near-native ability to process and produce formulaic language rapidly and appropriately (e.g., Forsberg 2010; Laufer \& Waldman, 2011). L2 learners are very challenged by formulaic language and develop facility with it very slowly, showing difficulty in understanding common usage and often using sequences that are first language-based and inappropriate.

There are many potential benefits to L2 learners mastering a range of formulaic sequences. They provide a nuanced, native-like quality to communication, they provide ready-made chunks of accurate language, and they allow for fluent speech through bypassing the constraints of cognitive load. Formulaic language is quite similar to vocabulary, and many sequences, especially collocations and complex verbs, have similar meanings to content words (e.g., Boers \& Lindstromberg, 2012, p. 84). Some types of sequences have discourse or social interaction functions - for example, idioms, exclamations, and pragmatic formulas. A significant body of work indicates that more use of formulaic sequences in speech or in writing is linked to higher assessments on speech and writing tasks (Boers, Eyckmans, Kappel, Stengers, \& Demecheleer, 2006; Dai \& Ding, 2010; Hsu \& Chiu, 2008; Keshavarz \& Samili, 2007). Experienced teachers notice that learners struggle with formulaic sequences and that very often they cannot interpret their meanings. Boers, Eychmans, and Stengers (2007) have shown that learners misunderstand figurative idioms despite context clues. Martinez and Murphy (2011) have discovered that learners often cannot give accurate meanings to formulaic sequences because they focus on the meanings of the individual lexical items that make up the sequence-Martinez and Murphy give the example It's about time and discuss how learners wrongly see about as a topic marker.

Not only do L2 learners have issues with interpreting formulaic language, they also have difficulty mentally processing formulaic language as native speakers do, and they miss the essential processing advantage of using them. Plenty of evidence shows that sequences held in memory act as ready-made stretches of language and a great shortcut in processing or formulating language. They help us to overcome the limitations of short-term memory and liberate our attention and energy to conceptualize and formulate other as- 
pects of discourse (see Wood, 2010, 2015, for an overview). Learners may understand that a sequence is formulaic, but that does not mean that they will mentally process it as formulaic. Clever psycholinguistic studies have provided evidence that learners process formulaic language faster than nonformulaic language, but that they process it more slowly than native speakers do (Columbus, 2010; Conklin \& Schmitt, 2008; Jiang \& Nekrasova, 2007). This may be partly due to the fact that native speakers have had endless opportunities to encounter even low-frequency sequences, while L2 learners have not (Ellis, Simpson-Vlach, \& Maynard, 2008). The figurative meanings of many formulaic sequences may also confuse things for learners - they frequently have to weigh literal and figurative options for a given sequence, taking up valuable reading, listening, and speaking time.

Obviously, dealing with formulaic language presents huge benefits and challenges to L2 learners. Over the years we have seen some classroom intervention studies (see Boers \& Lindstromberg, 2012, for a review) and some case studies of the efficacy of particular types of instruction, but it is clear that many issues around the teaching of formulaic language are still awaiting investigation. The collection of articles in this Special Issue of TESL Canada Journal represent one of the very few places to turn for some idea of how to deal with this essential component of language in classrooms.

The articles here fall into several categories of focus: teaching of formulaic language for spoken language; teaching of formulaic language for written language; specific approaches and methods of teaching formulaic language; and teaching of collocations.

There are two full-length articles here on teaching for spoken language. Both McGuire and Larson-Hall (pp. 1-25) and Thomson (pp. 26-53) address the role of formulaic sequences in L2 speech fluency and their teaching implications. It is heartening to see this important issue dealt with in research, as it has been a preoccupation of mine for many years (see Wood, 2010).

Two cleverly conceived and well-executed pieces of research on teaching formulaic language for writing are included here. Li and Volkov (pp. 54-75) examine the use of lexical bundles, a subset of formulaic language or multiword units, in tasks on a proficiency test. Murray (pp. 76-92) shows us a potentially valuable way to improve L2 academic writing proficiency with formulaic language. Both studies use innovative methods and present important results.

Two studies here address particular techniques or methods in teaching formulaic language. Koban Koç and Koç (pp. 93-110) examine the role of different media types on learning formulaic language. Le Thi, Rodgers, and Pellicer-Sánchez (pp. 111-139) look at the effects of explicit and textbook instruction of formulaic sequences in classroom contexts. Both studies take us a large step in the direction of better understanding how to select input and instructional strategies to improve learner facility with formulaic language. 
Two articles address issues around the teaching of collocations, a ubiquitous and elusive multiword phenomenon. Snoder (pp. 140-164) gives us a classroom-based experiment on the effects of several factors on improvement of learner production of collocations. Makinina (pp. 165-191) presents a study on factors influencing learner recognition of collocations. These two are sure to become go-to papers for anyone involved in teaching or researching collocations in language teaching.

Zavialova (pp. 192-204) presents an In the Classroom piece detailing a remarkable research- and theory-informed approach to teaching pragmatic formulas, with some tantalizing results from a case study.

In a Perspectives article, Szudarski (pp. 205-216) provides a remarkably crafted and useful overview of the state of the art on teaching and learning collocations.

This collection of articles is a great contribution to our knowledge of formulaic language in L2 language teaching. It is one of a very few to contain studies with this specific focus in one place, and stands to serve well all those who teach or research L2 language teaching. I commend the authors on their work and predict that these works will be often cited.

\section{David Wood}

\section{References}

Boers, F., Eyckmans, J., \& Stengers, H. (2007). Presenting figurative idioms with a touch of etymology: More than mere mnemonics? Language Teaching Research, 11(1), 43-62. https://doi. org/10.1177/1362168806072460

Boers, F., Eyckmans, J., Kappel, J., Stengers, H., \& Demecheleer, H. (2006). Formulaic sequences and perceived oral proficiency: Putting a lexical approach to the test. Language Teaching Research, 10(3), 245-261. https://doi.org/10.1191/13621688061r195oa

Boers, F., \& Lindstromberg, S. (2012). Experimental and intervention studies on formulaic sequences in a second language. Annual Review of Applied Linguistics, 32, 83-110. https://doi. org/10.1017/S0267190512000050

Columbus, G. (2010). Processing MWUs: Are MWU subtypes psycholinguistically real? In D. Wood (Ed.), Perspectives on formulaic language: Acquisition and communication (194-210). New York, NY: Continuum.

Conklin, K., \& Schmitt, N. (2008). Formulaic sequences: Are they processed more quickly than nonformulaic language by native and nonnativespeakers? Applied Linguistics, 29(1), 72-89. https://doi.org/10.1093/applin/amm022

Dai, Z., \& Ding, Y. (2010). Effectiveness of text memorization in EFL learning of Chinese students. In D. Wood (Ed.), Perspectives on formulaic language: Acquisition and communication (pp. 71-87). New York, NY: Continuum.

Ellis, N. C., Simpson-Vlach, R., \& Maynard, C. (2008). Formulaic language in native and secondlanguage speakers: Psycholinguistics, corpus linguistics, and TESOL. TESOL Quarterly, 42(3), 375-396. https://doi.org/10.1002/j.1545-7249.2008.tb00137.x

Forsberg, F. (2010). Using conventional sequences in L2 French. International Review of Applied Linguistics in Language Teaching, 48(1), 25-51. https://doi.org/10.1515/iral.2010.002

Hsu, J.-Y., \& Chiu, C.-Y. (2008). Lexical collocations and their relation to speaking proficiency of college EFL learners in Taiwan. Asian EFL Journal, 10(1), 181-204. 
Jiang, N., \& Nekrasova, T. M. (2007). The processing of formulaic sequences by second language speakers. Modern Language Journal, 91(3), 433-445. https://doi.org/10.1111/j.15404781.2007.00589.x

Keshavarz, M. H., \& Salimi, H. (2007). Collocational competence and cloze test performance: A study of Iranian EFL learners. International Journal of Applied Linguistics, 17(1), 81-92. https:// doi.org/10.1111/j.1473-4192.2007.00134.x

Laufer, B., \& Waldman, T. (2011). Verb-noun collocations in second language writing: A corpus analysis of learners' English. Language Learning, 61(2), 647-672. https://doi.org/10.1111/j.14679922.2010.00621.x

Martinez, R., \& Murphy, V. A. (2011). Effect of frequency and idiomaticity on second language reading comprehension. TESOL Quarterly, 45(2), 267-290.

Wood, D. (2010). Formulaic language and second language speech fluency: Background, evidence, and classroom applications. London, UK: Continuum.

Wood, D. (2015). Fundamentals of formulaic language: An introduction. London, UK: Bloomsbury.

\section{Un mot de l'éditeur invité}

Une quantité importante de différents types de recherche a porté sur les formules-des unités composées de plusieurs mots ayant un sens ou une fonction unitaire qui semblent préfabriquées et qui sont mémorisées et employées comme un seul mot. Étant donné que la nature, l'emploi et l'acquisition des formules font, depuis des années, l'objet de recherche, il est remarquable de constater le peu d'études portant sur les façons d'enseigner cet élément linguistique essentiel aux apprenants de langue seconde. Nous savons que seul les apprenants de L2 très avancés réussissent à traiter et à produire les formules rapidement et correctement (par ex., Forsberg 2010; Laufer \& Waldman, 2011). Les formules constituent un défi considérable pour les apprenants de L2; ceux-ci développent lentement une compétence relative à leur usage courant et emploient souvent des formules qui sont inappropriées et calquées sur leur première langue.

La maitrise d'un éventail de formules implique plusieurs bienfaits aux apprenants de L2. Ces expressions figées ajoutent une qualité de locuteur natif à la communication, offrent des petits paquets linguistiques précis et prêts à l'emploi et, en contournant les contraintes de la charge cognitive, favorisent la fluidité. Les formules s'apparentent au vocabulaire en ce qu'elles ont souvent des significations semblables aux mots lexicaux (p.ex., Boers \& Lindstromberg, 2012, p. 84). Certains types de séquences ont des fonctions discursives ou sociales - par exemple, les expressions idiomatiques, les exclamations et les formules pragmatiques. Un corpus important de recherche indique que l'utilisation élevée des formules à l'oral ou à l'écrit est liée à de meilleures évaluations aux tâches orales ou écrites (Boers, Eyckmans, Kappel, Stengers, \& Demecheleer, 2006; Dai \& Ding, 2010; Hsu \& Chiu, 2008; Keshavarz \& Samili, 2007). Les enseignants expérimentés remarquent que les étudiants luttent 
pour apprendre les formules et n'en comprennent pas souvent le sens. Boers, Eychmans et Stengers (2007) ont démontré que les apprenants comprennent mal les expressions idiomatiques, malgré les indices fournis par le contexte. Martinez et Murphy (2011) ont découvert que les apprenants n'arrivent souvent pas à proposer un sens précis aux formules puisqu'ils se concentrent sur le sens des mots individuels de la séquence.

Les apprenants de L2 n'ont pas seulement des problèmes à interpréter les formules, ils trouvent également difficile de traiter mentalement les expressions figées comme le font les locuteurs natifs et l'avantage crucial de s'en servir leur échappe. Une abondance de preuve démontre que les séquences mémorisées agissent comme segments linguistiques prêts à l'emploi qui constituent de superbes raccourcis dans le traitement ou la formulation du langage. Ils aident à surmonter les limites de la mémoire à court terme et libèrent notre attention et notre énergie pour nous permettre de concevoir et formuler d'autres aspects discursifs (voir Wood, 2010, 2015, pour un aperçu). Il se peut que les apprenants comprennent qu'une séquence constitue une formule, mais ils ne la traiteront pas forcément comme telle pour autant. De judicieuses études psycholinguistiques ont démontré que les apprenants traitent les formules plus rapidement que les phrases régulières, mais qu'ils le font plus lentement que les locuteurs natifs (Columbus, 2010; Conklin \& Schmitt, 2008; Jiang \& Nekrasova, 2007). Cela pourrait s'expliquer par le fait que les locuteurs natifs ont eu d'innombrables contacts avec mêmes les séquences les moins fréquentes, alors que ces occasions ne se seraient pas produites pour les apprenants de L2 (Ellis, Simpson-Vlach, \& Maynard, 2008). Le sens figuré de plusieurs formules pourrait également semer la confusion chez les apprenants, qui doivent souvent peser les options littérales et figurées pour une formule donnée, ce qui prend beaucoup de temps à l'oral, à l'écrit et à l'écoute.

De toute évidence, les formules présentent d'énormes avantages et de défis importants aux apprenants de L2. Au fil des années, des recherches ont porté sur des études d'intervention en salle de classe (voir Boers \& Lindstromberg, 2012, pour un aperçu) ainsi que sur des études de cas traitant de l'efficacité de certaines méthodes d'enseignement, mais il est clair que de nombreuses questions relatives à l'enseignement des formules restent à être étudiées. La collection d'articles dans ce numéro spécial de la Revue TESL du Canada représente un des seuls endroits où trouver des idées sur les façons d'adresser, en salle de classe, cette composante linguistique essentielle.

Les articles se rangent dans plusieurs catégories selon le thème: l'enseignement de formules pour la production orale; l'enseignement de formules pour la production écrite; approches et méthodes spécifiques pour l'enseignement des formules; et l'enseignement des expressions figées.

Deux articles de fond portent sur l'enseignement pour la production écrite. McGuire et Larson-Hall (pp. 1-25) d'une part et Thomson (pp. 26-53) d'autre part, traitent du rôle des formules dans la fluidité en L2 et des inci- 
dences qui en découlent pour l'enseignement. Il est encourageant de constater que la recherche se penche sur cette question importante, qui est une de mes préoccupations depuis plusieurs années (voir Wood, 2010).

Ce numéro comprend deux études habilement conçues et bien exécutées sur l'enseignement des formules pour la production écrite. Li et Volkov (pp. 54-75) examinent l'emploi d'expressions figées, une sous catégorie de formules, dans des tâches présentées dans un test de compétence. Murray (pp. 76-92) démontre une méthode prometteuse d'améliorer, par l'emploi de formules, la compétence en rédaction académique en L2. Ces deux études reposent sur des méthodes novatrices et elles présentent des résultats importants.

Deux articles traitent de techniques ou méthodes spécifiques pour enseigner les formules. Koban Koç et Koç (pp. 93-110) examinent le rôle de différents types de média sur l'apprentissage de formules. Le Thi, Rodgers et Pellicer-Sánchez (pp. 111-139) se penchent sur les effets d'un enseignement de formules en classe qui est explicit et basé sur un manuel. Ces deux études nous en apprennent beaucoup sur la sélection du contenu et des stratégies pédagogiques pour favoriser l'apprentissage des formules.

Deux articles traitent des enjeux de l'enseignement des expressions figées, ces phénomènes omniprésents et insaisissables. Snoder (pp. 140-164) décrit une expérience en salle de classe traitant des effets de plusieurs facteurs sur l'amélioration de la production d'expressions figées par des apprenants. Makinina (pp. 165-191) présente une étude portant sur les facteurs qui influencent la reconnaissance par les apprenants d'expressions figées. Ces deux articles deviendront assurément des références fréquemment consultées par tous ceux et celles qui sont impliqués dans l'enseignement ou la recherche des expressions figées en enseignement des langues.

Zavialova (pp. 192-204) présente un article dans la section En classe qui décrit une approche remarquable visant l'enseignement des formules pragmatiques, et qui repose sur la recherche et la théorie. Les résultats d'une étude de cas y sont présentés et s'avèrent intrigants.

Dans un article de la section Perspectives article, Szudarski (pp. 205-216) présente un aperçu remarquablement bien écrit et des plus utiles sur la situation actuelle de l'enseignement et l'apprentissage des expressions figées.

Cette collection d'articles contribuent grandement à notre connaissance des formules dans l'enseignement en L2. Une des seules à rassembler la recherche de ce domaine spécifique, elle est susceptible de s'avérer utile aux enseignants et aux chercheurs en enseignement de langues secondes. Je félicite les auteurs du travail accompli et je prévois que l'on fera souvent référence à leurs articles à l'avenir.

David Wood 